\title{
SELF CARE: JAPAN AND THE U.S. COMPARED
}

\author{
Marie R. Haug, ${ }^{1}$ Hiroko Akiyama, ${ }^{2}$ Georgeanna Tryban, ${ }^{3}$ Kyoichi Sonoda ${ }^{4}$ \\ and MAY WYKLE ${ }^{1}$ \\ 'Center on Aging and Health, Case Western Reserve University, Cleveland, OH 44106, U.S.A., \\ ${ }^{2}$ University of Michigan, Ann Arbor, MI 48109, U.S.A., ${ }^{3}$ Indiana State University, Terre Haute, IN \\ 47809, U.S.A. and 'University of Tokyo, Hongo, Bunkyo-ku, Tokyo, Japan
}

\begin{abstract}
Experience of common symptoms and subsequent self care behaviors among older adults are compared between Japan and the United States, two industrial countries with different cultural backgrounds and health insurance systems. Based on a modification of the Health Belief Model, perceived susceptibility to illness and belief in the efficacy of physician care were selected as major explanatory concepts for the decision to use self care for a complaint. Among 900 respondents in Japan and 728 in the United States, in three communities of varying size, self evaluations of good health, an indicator of low susceptibility, were very similar. Although Japanese respondents claimed fewer experiences of physician error, they still expressed lower preference for physician care than did those in the U.S. In addition, the Japanese reported far fewer symptoms than their U.S. counterparts during a three month period, and were more likely to use self care, even for symptoms they considered more serious. Disparate effects of such variables as good health behaviors, presence of a chronic condition and desire for autonomy are discussed in terms of cultural differences in the two countries.
\end{abstract}

Key words-elderly, self treatment, Japan, United States

\section{INTRODUCTION}

In all parts of the world, self care is the most common form of response to perceived symptoms of illness $[1,2]$. In the past, 'medicine without doctors' was widely practiced and still continues to characterize the preponderance of sickness care. Although intensive research into self care behaviors has begun recently [3-6], it is still a largely untapped area of health behavior and little is known about its content, range and determinants.

The definition of self care varies. The one adopted in many prior studies encompasses a broad range of activities that a lay person undertakes to maintain and promote health, and to prevent, detect, and treat health problems $[4,7,8]$. Although this multifaceted definition is useful in many ways, it is obvious that the behaviors bundled together (e.g. health promotion and self-treatment of symptoms), will have quite different determinants and consequences. Diffuseness in self care research, mainly due to this broad definition of self care, has hindered researchers in comparing and discussing their findings. Our definition of self care is much more specific. We define it in terms of response behavior to a perceived symptom without the involvement of physicians. Responses include deciding to do nothing, resting, taking over-the-counter medicine and resorting to various forms of self-treatment and care provided by lay persons, such as family members, without concurrent medical advice. It should be noted that although care provided by lay persons, for example taking chicken soup prepared by a family member for a cold symptom, is sometimes categorized in the literature as 'informal care' separately from 'self care', our definition includes all response behaviors to a symptom that do not involve a physician.
The theory underlying the analysis in this paper is that of the Health Belief Model $[9,10]$. This model, originally developed to explain preventive care, posits that perceived seriousness of an experienced symptom, perceived susceptibility to it, and belief in the efficacy of professional help will account for health behavior. Within the framework of the Health Belief Model, Haug and her associates developed a new model that was designed specifically to explain self care behavior, in the presence of signs of illness. For more information on the construction of the conceptual model, see the authors' earlier paper [5].

This model of self care postulates that self care action is explained by two major concepts. The first, preceived susceptibility, relates to the respondent's mental and physical health status and daily health practice. Individuals who perceive their health to be good and who are not plagued by psychological distress would have confidence in their own resilience and be ready to care for their ailments without resorting to professional help. Conversely, individuals with chronic conditions or who experience high numbers of symptoms could view themselves as basically in poor health and thus in need of a doctor's advice for any new ailment. In brief, positive health facilitates self care. Similarly individuals who are vigilant about their health, and who engage in good health practices with regard to diet and exercise, should be able also to distinguish between symptoms that require a physician's attention and those best treated by home remedies.

The second explanatory concept is the individual's attitude concerning the efficacy of physician care. This concept encompasses faith in doctors, claimed experience with medical error and self reliance in health care. The concept again borrows from the Health Belief Model, but in a reverse sense. Instead of belief in the benefits of normative action, namely 
consultation with a health professional, belief in the ineffectiveness of such action is substituted and triggers self treatment. In brief, negative views of physician care favor a choice of self care.

This model was developed in connection with an empirical study of self care among older adults that Wykle and Haug conducted in the United States [5]. The model was shown to account for self care decisions in response to symptoms perceived as mild as well as those viewed as serious, and to explain more variance than found in many studies using the Andersen's Health Behavior Model, once the effect of need was factored out. The study reported in the present paper was constructed to provide a replication in Japan of the Wykle and Haug study, but also to explore cultural differences in self care.

Cross-cultural studies of self care, particularly between a Western society and a non-Western society, are virtually non-existent. Caudill has noted that, "Culture and social structure are interrelated with the occurrence of disease and its treatment. This is true for both major and minor illnesses, and for the attitudes, beliefs and behaviors that make up the everyday care of the body" $[11$, p. 159]. The health behavior patterns and underlying principles identified in United States society may not be observed in other socio-cultural contexts. Comparative studies are essential to establish the generalizability of research findings and the validity of interpretations beyond a single culture. By conducting research in different cultures, we can often increase the range of variation in variables beyond that which is obtainable within a single setting, which enables a better understanding of the relationships among the variables. Consequently, it leads us to a much better sense of the robustness of research results [12].

The United States and Japan are leading Western and Eastern industrial societies, with very different historical backgrounds and belief systems. The two nations are similar in their basic economic structures, levels of industrialization, medical technologies, medical care organization and delivery systems, and epidemiological statistics. Like the United States, Japan is a capitalist, technologically advanced, urban, industrialized society. The population is stable, its general health standards are high, and the epidemiology of disease is similar to that in Western industrial societies [13]. In contrast with many European countries, but similar to the United States, the majority of medical care in Japan is delivered by health care providers in private practice. Furthermore, virtually all persons in Japan are covered by a national health insurance scheme, with medical care costs mostly reimbursed based on the fee-for-service system and people free to choose their health care providers. Japan's development, social structure and formal medical care system make Japan, except for the universal insurance feature, the most similar Asian society to compare with the United States.

In counterpoint to its developmental and structural similarities, Japan presents several contrasts that affect health behaviors. First, Japan has a unique medical history. Long ago Chinese medical practices were imported to Japan and flourished beside traditional folk practices [14]. In general, such Eastcrn health belicfs and practices attribute health prob- lems primarily to the imbalance in body homeostasis and focus their therapeutic efforts on restoring the balance by holistic methods. Self-reliance is encouraged in Eastern medicine which requires more active involvement of patients and their families in therapy compared to the Western medical model. Since Western medicine became the officially recognized and approved system of health care in 1883, it has gradually gained influence. Currently, partly because insurance coverage for non-Western medicine is quite limited, Western medicine is the dominant power in professional health care in Japan, although recent publicity concerning iatrogenic diseases related to synthetic drugs have eroded faith in the Western medical model. However, Eastern health beliefs and practices are still deeply rooted in Japanese health care, particularly in self care. Ohnuki-Tierney [15] offers an excellent discussion on medical pluralism in contemporary Japanese society.

A second counterpoint is the differing role of American and Japanese families in making health care decisions. Approximately two-thirds of older Japanese live with their adult children and only ten percent live alone. In comparison, as many as $40 \%$ of older urban Americans live in single person households [16]. Such differences in living arrangements affect the behavior of older persons in two primary ways. Family care, which in most cases is care by lay persons, is more readily available to people who live with family members than to those who live alone. For example, middle aged daughters-in-law usually undertake the major responsibility of house-work in Japanese multi-generation families. Under such living arrangements, a sudden illness of an older person would not affect his/her daily life as much as it would affect the life of a person who lives alone. On the other hand, living with a family could facilitate physician use, through the advice of relatives and their assistance in physical and financial access. These issues require empirical test.

All of these cultural differences are expected to affect personal health care decisions in the two countries. This paper describes similarities and variations in self care practices among older persons in the United States and Japan. More specifically, it addresses four questions:

(1) What are the differences between American and Japanese older persons in the experience of physical symptoms?

(2) What are the differences between American and Japanese older persons in the use of self care for such symptoms?

(3) What are the differences between the two countries in the factors that could account for the use of self care to deal with these symptoms?

(4) What are the differences between the two countries in the joint effects of these factors on the use of self care?

A comparison of the choices made by these older populations with respect to self care will test the generalizability of the conceptual model of self care beyond a single society, and, at the same time, offer clues to the social and cultural factors implicated in personal health care decisions. 


\section{METHODOLOGY}

\section{Overall model}

The conceptual scheme for the study is based on the Health Belief model. It diagrams the effect upon treatment action, namely self care practices, of perceived susceptibility to ill health and belief in physician efficacy, as modified by demographic controls (Fig. 1). The variables used to measure each concept are those for which congruent items were included in the data collection in Japan and the United States.

Socio-economic status is omitted from the model because it was considered impossible to rate occupations in a comparable fashion in the two countries. Fortunately education, useful as a surrogate for socio-economic status, could be measured identically in Japan and the U.S. Description of all measures is supplied below. Age, gender, education and social support were included as controls on the grounds that each has been shown to affect health behavior in the face of perceived illness. Consistent with the Health Belief model, the conceptual design includes perceived susceptibility to illness and belief in the utility of professional assistance as predictor variables, and differentiates resulting behavior on the basis of perceived seriousness of the complaint.

\section{Samples}

Answers to the research questions are derived from data collected in comparable surveys in these two countries. In the United States, 900 persons in three age groups (45-59, 60-74 and 75 and over), and in three communities of a Midwest state (major metropolis, midsized industrial city, and small rural town) were selected by random digit dialing. Following this computerized telephone process of identifying the random sample of subjects, face-to-face interviews were conducted, yielding an $81 \%$ response rate, with 728 cases for analysis. This study was completed in 1987. In Japan the data were collected in 1988 by contract with a major survey organization in Tokyo. A sample of 900 persons in the same age groups and community sizes were selected in the central part of the main island, by a process that involved random selection of a household from a stratified list of randomly selected neighborhoods in the comparative sized communities. Interviews were then secured from this household as a starting point until the quota from the neighborhood was attained. Thus absent persons and refusals were replaced on the basis of the randomly selected neighborhood and household. The procedure assured that a total of 900 interviews be completed, so that this number is available for comparative analysis. Major variables in the two studies were identical, with question wording congruent as assessed by back translation of the Japanese version.

\section{MEASURES}

\section{Dependent variables}

Self care rates. Each respondent was asked individually whether any of a list of 29 symptoms had been experienced in the past three months, and if so what action had been taken to deal with the symptom, including both self treatment and professional care. In the United States the first response was openended, followed by specific probes concerning the two types of care. In Japan, since the availability of open-ended response time was limited by the data collection agency, closed ended items based on these probes, were substituted. Thus a response that occurred in the United States, initially 'doing nothing' about a symptom, was not available in Japan. However it is reassuring that data from a subset of 124 respondents in Japan who kept daily health diaries for one month revealed that doing nothing as the sole response to a complaint was a very rare occurrence.

In both countries, respondents were also asked if they considered the symptom mild or serious. The symptom list contained complaints that fit potentially into either category. Among the less serious symptoms were running nose with fever, frequent sneezing, waking up stiff in the morning and upset stomach. Potentially serious symptoms included dizziness,

Perceived physician efficacy

Treatment action

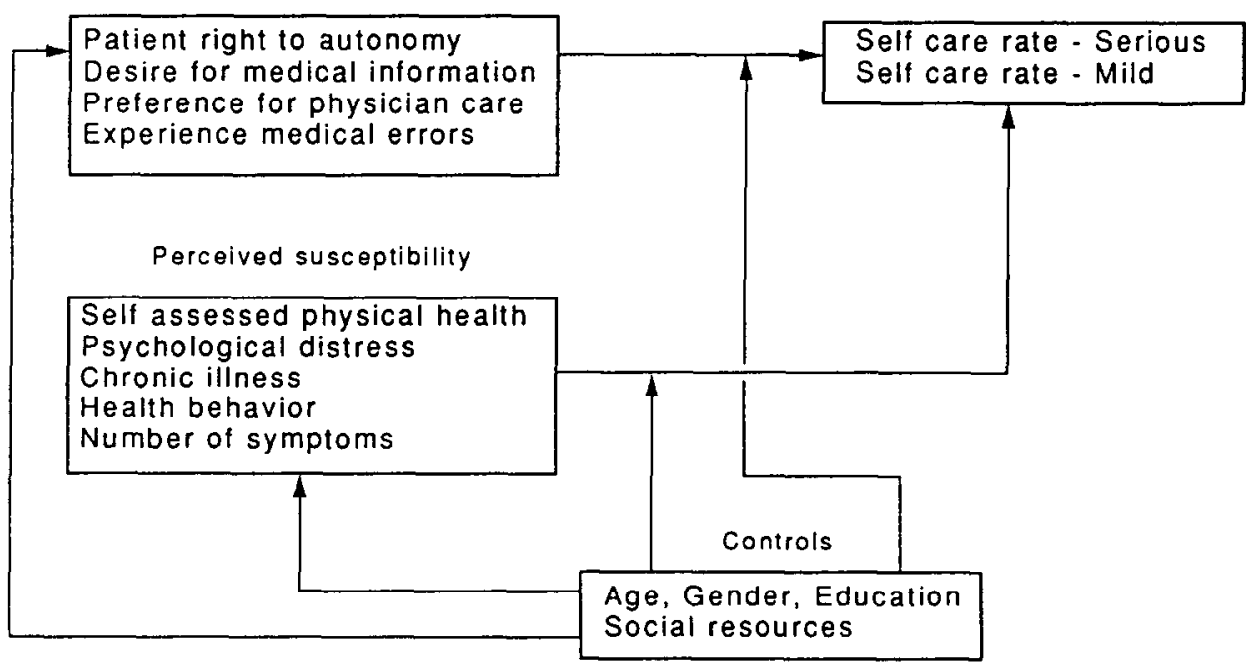

Fig. 1. Conceptual model for explaining self care practices. 
chest pains, shortness of breath after light activity and swelling of extremities. It will be noted that symptoms rather than diagnoses were listed, for example frequent sneezing rather than allergy [see Appendix for full list of symptoms].

Finally each individual reporting a symptom in Japan was also asked if he or she consulted a physician, either Eastern or Western, about the symptom. Self care rate for each respondent was then calculated by dividing the number of symptoms for which no physician was consulted by the total number of symptoms experienced. The results were placed in three categories for each respondent: all symptoms were responded to by physician utilization; all symptoms were responded to by self treatment; some symptoms were responded to with physician care while others received self treatment. This procedure was repeated separately for symptoms considered mild and those considered more serious. Since persons with multiple complaints often considered some mild and some serious, there was overlap in the results, so that mild and serious self care rate totals exceeded the total number of persons with symptoms.

\section{Independent variables}

Perceived physician efficacy. Nineteen items concerning attitudes to physicians and their care were factor analyzed using the data from Japan. The items were those developed in prior work by Haug and Lavin [17], augmented by selections from Krantz, Baum and Wideman [18]. Three factors emerged with eigenvalues greater than 1 . The first, entitled Right to Autonomy, with six items, encompassed statements concerning the right to make decisions about accepting treatment, to leave a hospital against advice, and the advisability of curing one's own illnesses. Reliability was 0.60. Preference for Physician Care, also including six items, involved confidence in physician care, the risks of eschewing medical advice, and the benefits of professional services. Alpha was 0.59. Finally, Desire for Medical Information, the third factor, contained seven items, three of which concerned the desire for full explanations of treatments and risks, as well as the utility of reading one's own medical records and securing views of more than one physician. The rationale for including this scale is that patients who secure information are in a better position to question their physician's recommendations and to act autonomously if those recommendations are considered ineffective. Cronbach's alpha was 0.65 .

The same items were factor analyzed earlier in the self care study in the United States, and produced different factors. Given that cross-national comparisons would only be possible with identical measures, the factor analysis results from the Japanese data were applied to the United States data, and are reported here. Although reliabilities for the resulting United States scales were considerably below the Japanese, the requirement of identical scales made it necessary to use them.

An additional indicator of belief in physician efficacy was reports of prior Experiences of Medical Error as evaluated by the respondent. Three items made up the scale; belief that there had been mistakes in care, that the physician had caused harm, and that care had come late. Reliability was 0.80 .

Perceived susceptibility. Five scales were used to measure this concept. Self Assessed Health was a summated scale, ranging from very poor to excellent (0-9). It combines assessment of own health in the last month, from poor to excellent, comparison of own health to that of age peers-worse, same or better-and extent to which health interfered with activities, from a great deal to not at all. Alpha reliability was 0.67 . Psychological Distress combined yes/no answers to seven items from the Langner scale into four categories from low (1) to very high (4) distress. The seven items were selected from those used in the Cleveland GAO study as non-somatic based on factor analysis [19]. They included difficulty in 'getting going', being a 'worrier', not feeling 'worthwhile', and being 'isolated'. Internal consistency reliability was 0.66 . Chronic illness was assessed as a dichotomy, with 0 measuring no chronic condition and 1 representing one or more conditions. The Health Behavior scale combined indicators of adequate sleep, eating breakfast daily and not snacking, low or no use of cigarettes and alcohol, and engaging in one or more physical activities, ranging from fishing to active sports. This scalc was originally developed by Berkman and Breslow [20] in the Alemeda County study, and has a bio-medical theoretical base. The elements of sleep, eating practices, substance use and exercise were considered to be cross-nationally relevant, since they did not include specific dietary elements that vary widely between countries. Note that good health behaviors do not necessarily correlate with self assessed good health. For example, sedentary drinkers and smokers who are not currently ill could believe themselves to be quite healthy. The scale was grouped into five categores with 1 equaling very poor and 5 very good Health Behaviors. Cronbach's alpha assessed reliability as 0.39 , suggesting the multi-dimensionability of the scale.

Total Number of Symptoms was also included as indicating perceived susceptibility, on the grounds that persons who had reported many conditions might consider themselves in fragile health, easily prey to becoming seriously ill from symptoms that the more robust could throw off. Accordingly resorting to self treatment might be considered risky.

\section{Controls}

Age was categorized in the three sampling groups: $45-59,60-74$, and 75 and over. Gender was a dichotomy, with female scored 0 and male 1 in the analysis. Education was trichotomized into less than 8 years of schooling, from 8 to 12 years or high school level, and more than 12 years of education or beyond high school. The Social Resources scale combined household composition, ranging from living alone, the least supportive, to living with spouse and children, the most supportive; frequency of contact with family and friends, availability of a confidante, loneliness, and availability of care if ill. This scale is an adaptation of the social resources section in the OARS [21]. The major revision involved type of contact with family or friends. Although frequency of telcphone contact questions were the same, queries 
about frequency of visits to others' homes were expanded for Japan, to include meeting with and going out together with others outside the home, since this is a more common form of social interaction in that country. Each element was coded from 0 (not supportive) to 3 (most supportive) and the seven elements summed. Responses, which ranged from scores of 1-21, were grouped into six categories, from very low support (score of 1-5) to very high (score of 19-21).

Analysis. Comparisons between the U.S. and Japan on all variables were evaluated by chi-square. The joint effects of the susceptibility and efficacy indicators on self care rates, as modified by the controls, were assessed by multiple regression. The rationale for this procedure is discussed further below.

\section{Findings}

The samples in the United States and Japan were not entirely similar demographically. In age groupings the United States had somewhat fewer of the very old, those 75 and above. Moreover in Japan, the procedures used produced a 50/50 male/female split, whereas in the United States close to two thirds of the respondents were women, reflecting both their predominance among the elderly and perhaps their greater willingness to be interviewed. In level of education, on the other hand, the two samples were virtually identical, with a third in both countries having more than the equivalent of a high school education. With respect to social support, the findings showed that in both countries the modal level was the next to the highest. Thirty-eight percent in the United States and $32 \%$ in Japan reported strong social support.

In response to the first research question on differences between the two countries in symptom experience, the results revealed the most marked difference in the percentage of respondents who claimed they had experienced none of the 29 listed symptoms. It will be recalled that the list included common complaints like cold symptoms or stomach upsets, as well as potentially more serious problems such as dizziness and chest pains. Only $10 \%$ of the United States respondents reported experiencing none of the symptoms in a prior three month period, compared to $47 \%$ of the Japanese with no reported complaints (Table 1).

The fear that this disparity was due to language differences in the instruments was allayed by the fact that the Japanese data were similar to those found in

Table 1. Number of symptoms experienced in a three month period, based on a list of 29 , comparing the United States and Japan

\begin{tabular}{lcc}
\hline & $\begin{array}{c}\text { U.S. } \\
(N=728) \\
\%\end{array}$ & $\begin{array}{c}\text { Japan } \\
(N=900) \\
\%\end{array}$ \\
\hline None & 10 & 47 \\
1 or 2 & 26 & 38 \\
3 or 4 & 21 & 10 \\
5 or 6 & 15 & 3 \\
7 or 8 & 11 & 1 \\
9 or more & 18 & 1 \\
Total & 101 & 100 \\
Total symptoms reported & 3577 & 1274 \\
\hline
\end{tabular}

$\chi^{2}$, df $5=520.89, P<0.0000, V=0.57$
Table 2. Differences in reported symptom frequency for ten common complaints, comparing the United States and Japan

\begin{tabular}{lcc}
\hline & $\begin{array}{c}\text { Percentage with reported } \\
\text { symptom of those with } \\
\text { any symptom }\end{array}$ \\
& $\begin{array}{c}\text { U.S. } \\
\%\end{array}$ & $\%$ \\
\hline & & \\
& 9 & 11 \\
\hline A. Japan rate higher than U.S. & 27 & 32 \\
$\quad$ Cold signs for two days & & \\
$\quad$ Frequent backaches & 25 & 9 \\
B. U.S. rate at least 25\%, and & 26 & 19 \\
higher than Japan & 29 & 11 \\
$\quad$ Shortness of breath & 29 & 7 \\
Insomnia & 30 & 5 \\
$\quad$ Joint or muscle pain & 37 & 4 \\
Memory problems & 40 & 17 \\
Swelling of arms, legs, feet & 40 & 8 \\
Nervousness & & \\
Waking up exhausted & Waking up stiff &
\end{tabular}

national government surveys of health in that country, and were consistent with results on studies of other complaints (e.g. Lock [22]). Moreover, those who claimed to have experienced a symptom were much more likely to have reported multiple ailments in the United States than in Japan. Forty-four percent of those in the United States reported five or more complaints compared to only $6 \%$ of those in Japan. In the United States 3577 symptoms were reported by 655 persons (an average of 5.5) compared to 1274 in Japan for 475 persons (an average of 2.7 ).

The pattern of symptom experience was diverse as well. Among the 29 complaints about which the subjects were interviewed only two showed a higher incidence over three months in Japan than in the United States. Cold symptoms lasting at least two days were reported by $9 \%$ in the United States and $11 \%$ in Japan, while frequent backaches occurred among $27 \%$ in the United States and $32 \%$ in Japan. In all other cases, the United States showed higher rates than Japan (Table 2). Among the eight complaints that $25 \%$ or more respondents reported in the United States, only insomnia showed a rate near that in Japan. The most marked disparity was in reported nervousness 37 vs $4 \%$ in the two countries, and in waking up stiff in the morning, 40 vs $8 \%$.

Behaviors in response to symptoms were also markedly different. The answer to question two regarding self treatment showed that among those who had experienced symptoms, $50 \%$ of the Japanese used self care only, compared to only $27 \%$ of those in the United States who relied exclusively on nonprofessional treatment (Table 3). In Japan, use of both Eastern and Western practitioners was counted as physician care, since doctors of both persuasions are accredited as medical professionals.

Because a person's beliefs about the seriousness of a symptom could affect his or her treatment actions, rate of self care in connection with symptoms perceived as serious was compared with symptoms perceived as mild. The results for symptoms believed to be only mild again revealed a higher rate of self care in Japan than in the United States. Nearly two-thirds of persons in Japan relied entirely on self treatment if they considered their symptom mild, while in the United States only about a third with the same symptom evaluation did so (Table 4A). 
Table 3. Rate of self care for 29 symptoms comparing the U.S. and Japan

\begin{tabular}{lcc}
\hline & $\begin{array}{c}\text { U.S. } \\
(N=657) \\
\%\end{array}$ & $\begin{array}{c}\text { Japan } \\
(N=475) \\
\%\end{array}$ \\
Self care rate & 11 & 26 \\
\hline No self care (100\% MD use $)$ & 62 & 24 \\
Mixed self care and MD use & 27 & 50 \\
Self care only & 100 & 100 \\
Total & &
\end{tabular}

Differences were somewhat less marked with respect to symptoms the respondents evaluated as more serious. In these circumstances $24 \%$ of the United States persons compared to $31 \%$ of the Japanese relied solely on self treatment (Table 4B).

The factors considered to account for self care, as posed in research question three involve both belief in physician efficacy and in one's own susceptibility to illness. Ideas about the right to autonomy in doctor-patient relationships, patients' desire for medical information, preference for physician care when ill, and experience of medical error are viewed as four indicators of perceived physician efficacy. It should be noted in presenting these and the variables assessing respondents' health, frequencies are provided only for those who claimed symptoms. However their characteristics did not differ significantly from those of the total number of respondents.

With respect to belief in patient autonomy, some older adults in Japan were a bit more likely than those in the U.S. to think they had little right to an autonomous relationship with a physician (15 vs $8 \%$ ). The implication is that lay persons are less able than professionals to deal with illness, and should defer to their greater skill (Table 5A). However about half in each country were on the higher end of this distribution, demonstrating a desire to maintain patient rights. The fact that differences by country are statistically significant is due more to the size of the samples than the size of the differences.

Further in Japan a similar minority of older adults with symptoms were less likely to be interested in sccuring information on thcir condition than those in the United States, $20 \%$ in Japan in the lowest information seeking categories, compared to $2 \%$ in these

\section{Table 4}

A. Rate of self care for symptoms considered mild comparing the U.S. and Japan

\begin{tabular}{lcc}
\hline & $\begin{array}{c}\text { U.S. } \\
(N=632) \\
\%\end{array}$ & $\begin{array}{c}\text { Japan } \\
(N=393) \\
\%\end{array}$ \\
Self care rate & 11 & 19 \\
\hline No self care (100\% MD use $)$ & 54 & 18 \\
Mixed self care and MD use & 34 & 64 \\
Self care only & 99 & 101 \\
\hline Total & \\
\hline
\end{tabular}

$\chi^{2}$, df $2=135.02, P<0.000, V=0.36$.

B. Rate of self care for symptoms considered more serious comparing the U.S. and Japan

\begin{tabular}{lcc}
\hline Self care rate & $\begin{array}{c}\text { U.S. } \\
(N=302) \\
\%\end{array}$ & $\begin{array}{c}\text { Japan } \\
(N=173) \\
\%\end{array}$ \\
\hline No self care (100\% MD use $)$ & 43 & 56 \\
Mixed self care and MD use & 33 & 13 \\
Self care only & 24 & 31 \\
Total & 100 & 100 \\
\hline
\end{tabular}

$x^{2}, d f 2=28.86, P<0.0000, V=0.25$.
Table 5

A. Belief in patients' right to autonomy: United States and Japanese elderly with symptoms

\begin{tabular}{lcc}
\hline & $\begin{array}{c}\text { U.S. } \\
(N=654) \\
\%\end{array}$ & $\begin{array}{c}\text { Japan } \\
(N=451) \\
\%\end{array}$ \\
\hline Low $(0,1)$ & 8 & 15 \\
Moderate $(2,3)$ & 42 & 37 \\
High $(4,5)$ & 43 & 40 \\
Very High $(6)$ & 7 & 8 \\
\hline
\end{tabular}

$\chi^{2}$, df $3=16.06, P<0.005, V=0.12$.

B. Belief in medical information seeking: United States and Japanese elderly with symptoms

\begin{tabular}{lcc}
\hline & $\begin{array}{c}\text { U.S. } \\
(N=653) \\
\%\end{array}$ & $\begin{array}{c}\text { Japan } \\
(N=475) \\
\%\end{array}$ \\
\hline Low $(0,1,2)$ & 2 & 20 \\
Moderate $(3,4)$ & 50 & 32 \\
High $(5,6)$ & 46 & 38 \\
Very High $(7)$ & 2 & 10 \\
\hline
\end{tabular}

$x^{2}$, df $3=154.94, P<0.0000, V=0.37$.

categories in the United States (Table 5B). Again it is noteworthy that $48 \%$ in each country have a high or very high belief in the right to information. This attitude is consistent with adherence to autonomy $v i s-\grave{a}-v i s$ the physician, since knowledge of one's condition is an asset in dealing on an equal footing with a medical professional.

In the United States, symptomatic respondents were more likely to prefer a physician's services when ill: $23 \%$ strongly preferred this care compared to $14 \%$ in Japan (Table 6A). Again a sizable minority, from 24 to $32 \%$ were not so sure they wanted medical attention.

These preferences existed in the face of the fact that persons in the United States were much more apt to claim having experienced a physician making an error in their care, than were persons in Japan $(46 \%$ compared to $26 \%$ ) (Table $6 \mathrm{~B}$ ). Whether this finding reflects higher quality of care in Japan, or a tendency of Americans to complain about their medical outcomes cannot be determined from these self-reports.

The second set of factors that could account for turning to self care were entitled perceived susceptibility and included both mental and physical health, as well as commitment to good health practices,

Table 6

A. Preference for physician care when ill: United States and Japanese elderly with symptoms

\begin{tabular}{lcc}
\hline & $\begin{array}{c}\text { U.S. } \\
(N=648) \\
\%\end{array}$ & $\begin{array}{c}\text { Japan } \\
(N=475) \\
\%\end{array}$ \\
\hline Low $(0,1)$ & 4 & 5 \\
Moderate $(2,3)$ & 20 & 27 \\
High $(4,5)$ & 53 & 55 \\
Very high (6) & 23 & 14 \\
\hline
\end{tabular}

$\bar{\chi}^{2}$, df $3=19.24, P<0.000, V=0.13$.

B. Perceived physicians' past errors: United States and Japanese elderly with symptoms

\begin{tabular}{lcc}
\hline & U.S. & Japan \\
& $(N=655)$ & $(N=460)$ \\
$\%$ & $\%$ \\
\hline None & 54 & 74 \\
Some & 46 & 26 \\
\hline
\end{tabular}

$\chi^{2}, d f 1=45.40, P<0.0000, V=0.20$. 
Table 7

A. Level of psychological distress: United States and Japanese elderly with symptoms

\begin{tabular}{lcc}
\hline & $\begin{array}{c}\text { U.S. } \\
(N=655) \\
\%\end{array}$ & $\begin{array}{c}\text { Japan } \\
(N=473) \\
\%\end{array}$ \\
\hline Low (1) & 32 & 43 \\
Moderate low (2) & 44 & 39 \\
High (3) & 16 & 14 \\
Very high (4) & 9 & 4 \\
\hline
\end{tabular}

$\chi^{2}$, df $3=22.81, P<0.000, V=0.14$.

B. Self assessed health: United States and Japanese elderly with symptoms

\begin{tabular}{lcc}
\hline & $\begin{array}{c}\text { U.S. } \\
(N=655) \\
\%\end{array}$ & $\begin{array}{c}\text { Japan } \\
(N=474) \\
\%\end{array}$ \\
\hline Very Poor $(0,1,2)$ & 10 & 9 \\
Poor $(3,4)$ & 10 & 12 \\
Fair $(5,6)$ & 27 & 25 \\
Good $(7,8)$ & 42 & 45 \\
Excellent $(9)$ & 11 & 9 \\
\hline
\end{tabular}

$\chi^{2}, d f 4=3.70, \mathrm{~ns}, V=0.06$.

presence of one or more chronic conditions, and frequency of symptom reporting. These could indicate a person's perceived low likelihood of getting worse and thus willingness to forego reliance on professional help. On the measure of psychological distress, $43 \%$ of these Japanese older adults reported little or no distress, compared to $32 \%$ of the United States respondents, and in the same vein $25 \%$ in the United States elders admitted to high or very high distress, compared to $18 \%$ of the Japanese (Table 7B). These results suggest more psychological well-being among the Japanese than the American respondents.

With respect to the scale combining three facets of self assessed physical health, evaluations were about equal. Fifty-four percent of those in Japan considered their health very good or excellent, compared to $53 \%$ of those in the United States with the same view (Table 7A). However, not consistent with these findings, respondents in the two countries were dissimilar with respect to the presence of chronic conditions. About a third reported one or more chronic ailments in Japan, but in the U.S. two-thirds claimed such conditions (Table 8A). The lower rate in Japan matches the lower incidence of experienced symptoms in that country as compared to the United States.

The index of Good Health Behavior, which combined indicators of adequate sleep, reasonable diet, low use of alcohol and cigarettes, and physical exercise, suggested an orientation to maintaining one's good health. On this measure, the United States
Table 8

A. Prevalence of chronic conditions

\begin{tabular}{lcc}
\hline & $\begin{array}{c}\text { U.S. } \\
(N=655) \\
\%\end{array}$ & $\begin{array}{c}\text { Japan } \\
(N=475) \\
\%\end{array}$ \\
\hline Chronic condition & 33 & 64 \\
Some & 67 & 36 \\
Total & 100 & 100 \\
\hline
\end{tabular}

$\chi^{2}, d f 1=108.12, P<0.0000, V=0.31$.

B. Good health behaviors: United States and Japanese elderly with symptoms

\begin{tabular}{lcc}
\hline & $\begin{array}{c}\text { U.S. } \\
(N=657) \\
\%\end{array}$ & $\begin{array}{c}\text { Japan } \\
(N=475) \\
\%\end{array}$ \\
Health behavior & 4 & 3 \\
Very poor 1 & 11 & 14 \\
2 & 21 & 31 \\
3 & 33 & 35 \\
4 & 32 & 17 \\
Very good 5 & 101 & 100 \\
Total & &
\end{tabular}

$\chi^{2}$, df $4=36.39, P<0.0001, V=0.18$

outshone the Japanese respondents, with $32 \%$ reporting very good behavior, compared to only $17 \%$ in Japan (Table 8B).

Also used as an indicator of health status was the number of symptoms reported in the prior three months. The rationale is that persons with more symptoms are likely to consider their health fragile. As reported above, persons in Japan claimed fewer symptoms than those in the United States.

To determine the answer to question four, concerning Japanese and American differences in the joint effects of attitudes toward physician efficacy and beliefs in own mental and physical health on use of a self care, multiple regression was employed, controlling for age, gender, education and social resources. The trichotomous outcomes, rates of self care, are not normally distributed, it will be noted, but ordinary least squares are considered robust in the face of departures from normality. Also factors reaching statistical significance at the 0.10 level are reported, as being indicative of trends in the data. Since persons reporting multiple symptoms could have considered some mild and some serious, the variable of symptom seriousness could not be used in the regression, because it did not apply exclusively to some subjects. Accordingly separate regressions were run with self care for symptoms considered mild and for symptoms considered more serious, as two distinct outcomes, along with a regression for the total set of symptoms, which perforce omitted seriousness as a measure.

Table 9. Regression of self care rate for all symptoms on health and attitude variables, with demographics controls, comparing the U.S. and Japan

\begin{tabular}{lcccc}
\hline & \multicolumn{2}{c}{ U.S. } & \multicolumn{2}{c}{ Japan } \\
& $\beta$ & $P^{\mathrm{a}}$ & $\beta$ & $P^{2}$ \\
\hline Chronic condition & 0.072 & $<0.09$ & -0.129 & $<0.01$ \\
Good health behavior & -0.076 & $<0.06$ & -0.102 & $<0.03$ \\
Self assessed health & 0.094 & $<0.06$ & 0.127 & $<0.02$ \\
Total number of symptoms & -0.086 & $<0.07$ & - & - \\
Desire for information & - & - & 0.081 & $<0.08$ \\
Social resources & - & - & -0.078 & $<0.10$ \\
Education & -0.089 & $<0.03$ & - & - \\
$\quad$ Adjusted $R^{2}$ & \multicolumn{2}{c}{0.03} & & \multicolumn{2}{c}{0.05} & \\
& $F_{9,642}=5.49, P$ & $<0.001$ & $F_{9.445}=5.67, \quad P<0.0001$ \\
\hline
\end{tabular}

${ }^{2}$ Probabilities of $<0.10$ are reported to indicate trends in the data. 
Tabie 10. Regression of self care rate for symptoms considered mild on health and attitude variables, with demographics controls, comparing the U.S. and Japan

\begin{tabular}{|c|c|c|c|c|}
\hline \multirow{4}{*}{$\begin{array}{l}\text { Total number of symptoms } \\
\text { Chronic condition }\end{array}$} & \multicolumn{2}{|c|}{ U.S. } & \multicolumn{2}{|c|}{ Japan } \\
\hline & \multicolumn{2}{|c|}{$(N=624)$} & \multicolumn{2}{|c|}{$(N=373)$} \\
\hline & -0.139 & $<0.01$ & - & - \\
\hline & 0.073 & $<0.09$ & -0.142 & $<0.01$ \\
\hline Psychological distress & 0.095 & $<0.05$ & - & - \\
\hline Education & -0.068 & $<0.10$ & - & - \\
\hline Right to autonomy & - & -- & 0.10 & $<0.07$ \\
\hline Adjusted $R^{2}$ & \multicolumn{2}{|c|}{$5.19, P<0.001$} & \multicolumn{2}{|c|}{$F_{3.370}=5.04, P<0.01$} \\
\hline
\end{tabular}

"Probabilities of $<0.10$ are reported to indicate trends in the data. In the U.S. Health Status entered at the 0.14 level, while in Japan Right to Information entered at the 0.12 level.

Explanatory patterns showed both similarities and differences between the two countries. In both the United States and Japan, factors related to self care use for the total set of symptoms included better self assessed health, and fewer good health behaviors although technically these factors qualified only as trends in the United States. In Japan absence of a chronic condition encouraged self treatment while in the United States a trend toward presence of chronic ailments related to self care, as did a trend to fewer perceived symptoms. Only one attitude variable, desire for information, was relevant to self care, but it was limited to a trend in Japan. Also, in Japan, fewer social resources were related to self treatment. Lower education was the most significant predictor of self care in the United States sample, but had no effect in Japan (Table 9). Explained variance in self care rate was minimal; $R^{2}$ was $3 \%$ in the U.S. and $5 \%$ in Japan respectively. But it should be noted that these results did not include a variable of 'need', and in this respect explained variance was similar to that remaining in utilization studies after 'need' has been ruled out (e.g. Ref. [23]).

Regression results for symptoms believed to be mild exhibited less cross-national congruence. Chronic condition, the only common explanatory factor was again opposite in effect; absence of chronicity was significant in Japan, in contrast to a trend for presence of chronicity fostering self care in the United States. Fewer symptoms experienced and greater psychological distress along with lower education related to self care rate in the United States, but only belief in the right to autonomy had a similar effect in Japan. Again explained variance was small, 3 and $4 \%$ respectively (Table 10).

As for symptoms believed to be serious, only less good health behavior among the Japanese respondents, and lower preference for physician care among the United States respondents had any effect on their self care rates. Moreover the explained variance was trivial, $1 \%$ in the U.S. and $2 \%$ in Japan (Table 11).

\section{DISCUSSION}

In response to research questions one and two, concerning cross-cultural differences in symptom experience and self care behavior, the findings show that older adults in Japan, as compared to those in the United States, report fewer symptoms and are more likely to use self treatment for perceived symptoms, even if they are considered more serious. The most meaningful variations in attributes that might explain these diffcrences, as posed in question three, are the Japanese lower rate of emotional ill-health, lower desire for health information, and less preference for physician care, despite fewer claimed experiences of physician error. On the other hand, older adults in the two countries were almost identical in evaluations of their own state of health, although in the Japanese society the respondents were less likely to engage in good health practices.

In answer to question four about the joint effect of these variables, results revealed that different sets of factors explain the use of self care in these two countries. In the United States fewer symptoms and lower education were related to more use of self care, while greater desire for information and fewer social resources applied only to Japan. This lends some support to the suggestion that family involvement facilitates physician utilization, in Japanese culture. The result is intriguing, and the role that social resources play in self care needs to be explored in future studies. Japan and the United States shared three explanatory factors. In both countries persons with higher self-assessed health more frequently relied on self care. In contrast, it was also those persons who practiced fewer good health behaviors who used more self care. Finally, although the presence of

Table 11. Regression of self care rate for symptoms considered more serious on health and attitude variables, with demographics controls, comparing the U.S. and

\begin{tabular}{|c|c|c|c|c|}
\hline & \multicolumn{2}{|c|}{ U.S. } & \multicolumn{2}{|c|}{ Japan } \\
\hline & \multicolumn{2}{|c|}{$(N=297)$} & \multicolumn{2}{|c|}{$(N=166)$} \\
\hline \multirow{3}{*}{$\begin{array}{l}\text { Good health behavior } \\
\text { Preference for } \mathrm{MD} \text { care } \\
\text { Adjusted } R^{2}\end{array}$} & - & - & -0.17 & $<0.03$ \\
\hline & -0.10 & $<0.10$ & - & - \\
\hline & \multicolumn{2}{|c|}{$\begin{array}{c}0.01 \\
F_{1.296}=2.86, P<0.10\end{array}$} & \multicolumn{2}{|c|}{$\begin{array}{c}0.02 \\
F_{1,165}=5.01, P<0.03 \\
\end{array}$} \\
\hline
\end{tabular}

${ }^{2}$ Probabilities of $<0.10$ are reported to indicate trends in the data. 
chronic conditions had a significant impact on self care, the variable expressed itself in opposite directions in the two cultures. In the United States the presence of chronic conditions led to more self care, but in Japan the opposite was true.

For mild symptoms, in the United States less psychological distress was explanatory, while a desire for autonomy affected use of self care in Japan. For serious symptoms, only low preference for M.D. care was effective in the United States, while in Japan poor health behavior was explanatory. The low amount of explained variance for total, mild and serious symptoms reveals that many explanatory factors were missing in the research. Furthermore, there are apparent contradictions in the data. The presence of a chronic condition fosters self care in the United States, but its absence relates to self care in Japan. The Japanese report fewer symptoms but engage in less good health behavior, and they use both more physician care and more self care than found in the United States data.

The disparate effect of chronic illness may well be a rcsult of cultural differences. In the United States the need to cut health costs, particularly among the elderly and the focus on patient responsiblity for the management of chronic illnesses, along with an increasing emphasis on consumerism [17] argues for self treatment. In Japan the presence of a chronic condition may be decisive in drawing the individual into the network of repeat visits and monitoring that is characteristic of the Japanese biomedical system [15]. On the other hand, being free of a chronic ailment, in terms of the Health Belief model, indicates confidence in ones low susceptibility to disease and thus assurance that self treatment will work.

The Japanese place a high priority on health and medical care and they report themselves as being in good health both objectively and subjectively [24]. The research reported here confirms this finding and shows that the Japanese, compared to a U.S. sample, report fewer symptoms in a three month retrospective, but in apparent contradiction claim less good health behavior. The contradiction can be resolved through the recognition that the effects of poor dietary habits, indulgence in cigarettes and alcohol and lack of exercise do not necessarily result in immediate ill health, even if they have a negative impact on cardiovascular disease and cancer in the long run. If anything, the report of poor health behavior appears incompatible with personal autonomy, the decision to take charge of ones own health and well-being, a disparity that is discussed further below. The greater use of both physician care and self care in Japan than in the United States depending on the seriousness of a condition, is not really an anomaly, but instead indicative of a focus on good health that is more central to the culture of Japan than it has been in the United States. The Japanese are very sensitive to the condition of the body, and the management of its care [11] and will selectively use professional and/or lay care as they consider appropriate for a specific bodily change.

One line of logic argues that good health results from speedy professional attention to any symptoms of ill health. This reasoning would lead us to expect our Japanese sample to report high rates of physician utilization for the symptoms they did experience. As already noted above, Japan has a national health care system that, since 1961, has covered its entire population at a relatively low cost to the consumer. In addition, its urban areas, in particular, are well supplied with physicians, making ambulatory care at local clinics easy to obtain [25]. Furthermore, Lock [26] argues convincingly that the medical institution and its medicalization of (especially women's) socially induced psychosomatic problems serves to satisfy important non-medical needs. Secondly, the cultural pattern of recommended or required revisits is present, due primarily to the financial rewards of over-scheduling patients [15]. Indeed the rate for those receiving medical care has been increasing, though this is due in part to the aging of the population.

On the other hand, the national health care system is unlikely to account for the much lower rate of reported symptoms in Japan. One speculation is that the Japanese are in fact healthier, as evidenced by their lower prevalence of chronic conditions, and their self assessed emotional well being, as well as government statistics on symptom prevalence [27]. A counter view is that older Japanese are more reticent about admitting to psychological distress or ill health, or may have a different conception of when a bodily change can be considered a symptom.

The Japanese, when compared to Americans, do show a higher rate of exclusive physician consultation. However, this is coupled with an even larger reliance on self care. This occurs in spite of the fact that Americans and Japanese report similar preferences for physician care and even fewer instances of physician error in Japan. In other words, Japanese, unlike Americans, seem to do one or the other.

One key to this pattern lies in the doctor-patient relationship in Japan, which, as is true of all countries, is embedded in culture, and thus embodies aspects not present in the American relationship. It is highly non-verbal $[15,26]$ and hierarchical $[25,28]$, and may be subject to manipulation, as for example the use of gift giving by patients to establish a reciprocal, not wholly dependent relationship with the physician [29, p. 41]. Patients do not expect to be equal participants, or involved in health care decision-making. Indeed this has led to growing patient dissatisfaction, but without the possibilities for change through confrontation that exist in the consumer movement in the U.S. [26]. This acceptance of the cultural definition of the role of the physician can be seen in the data on information seeking. This would seem to indicate a low desire for knowledge about one's medical condition or health. However, the Japanese are avid consumers of medical information [26]. A deconstruction of the scale coupled with cultural knowledge suggests a different conclusion: that the scale accurately reflects the Japanese acceptance of cultural norms prohibiting discussion as indicative of lack of faith and respect that could even put the continued relationship with the physician in jeopardy [25]. This leads to the conclusion that due to their recognition of the loss of autonomy once the hierarchical doctor-patient relationship is entered, the Japanese prefer to avoid use of 
physicians except in response to the more serious symptoms. This allows them the autonomy of self care through published materials and over the counter and kanpo medications that flourish in Japan [30]. Only if the symptom is deemed really serious, and the patients feel their lay knowledge is inadequate, are they willing to forego autonomy, and place themselves fully in the hands of the doctor [31].

Although there is a statistically significant difference favoring patients' rights in the U.S., as pointed out in the results section, this significance is primarily an artifact of the large sample size, since about half in each country support the value of patient autonomy in dealing with physicians. In fact the degree of similarity of Japanese and U.S. views on autonomy is arresting given the established physician belief that patients have no 'right' to know their diagnosis and prognosis in the case of terminal illness [32], and should be protected from such knowledge by their physician. Debate on the ethics of such denial of information was intensified by the emperor's death in 1990 from cancer, and the controversy about the non-disclosure of his diagnosis, and has continued with his cousin's insistence in 1991 on disclosure of his cancer diagnosis.

A close examination of the nuances of the items in the Right to Autonomy Scale shows that it contains references to the level of autonomy a person has in decision making once the physician-patient relationship is established as well as the person's right to terminate that relationship. Thus autonomy may express itself in two ways. One dimension is patients belief or perception of the level of autonomy that will be afforded them, or that they can legitimately expect, ... an expectation that as Antonovsky [29] shows is at least in part culturally based. The cultural factors that produce this particular role definition will influence the patients' perception of the appropriate role behaviors and rewards from the autonomy within that role. In Japan, noted for its emphasis on hierarchical relationships in general and in the medical profession as a mirror of society [28], the physician-patient relationship is seen as one in which one's dependency must be expressed, making it simultaneously opportunity and obligation [25].

A second dimension of autonomy, that was not originally conceptually distinct but whose distinction is urged by the results, is personal autonomy as the rejection of passivity in regard to the body's health. This involves the individual's awareness of physical states and response to his/her symptoms, and it stands in contrast to the relational autonomy that one experiences within interpersonal relationships. While relational autonomy is low in the physician-patient context there is support in this study as well as others $[14,15,26,27]$ that personal autonomy in regard to health is high. This distinction may help to explain the autonomy and self care findings from these data.

An apparent contradiction to personal autonomy is the lower score on good health behaviors, particularly smoking and drinking alcohol, to which the Japanese would be expected to adhere. However, the negative effects of neglecting good health behaviors have only recently been publicized, and health education for prevention, beyond the areas of cancer and hypertension, is not yet well coordinated or widespread [25]. In practical terms the Japanese already practice an important good health behavior, walking, which no doubt contributes to the lower level of some of their particular symptoms, such as shortness of breath, stiffness, and edema of the legs. Activity in the form of walking is an integral part of Japanese daily life as well as the most popular sports activity for the elderly [33]. In contrast to American society, Japan's reliance on public transportation and daily food shopping at a local market necessitate a greater amount of routine daily walking, not for sport but as transportation to the bus stop and market. Furthermore, many Japanese elderly still prefer to sit on a tatami floor rather than a Western style sofa [33] and this, combined with the use of squat toilets and lower incidence of obesity, results in Japanese with greater physical flexibility. On the other hand, the different seasons in which interviewing was done (January-March in Japan and July-September in the United States) can account for the slightly higher incidence of colds in the Japanese population.

Another pathway that could be explored in future research on Japan concerns differences between those who report symptoms, and those who do not. Data not reported here show that those who do not claim to have experienced symptoms are no different from the symptomatic in attitudes toward autonomy, physician care and the desire for medical information. But they are less likely to have a chronic condition and to report psychological distress. Comparisons between the symptomatic and asymptomatic in the United States were not possible because, unlike the situation in Japan, nearly everyone reported having experienced a complaint. Differences in defining bodily changes as symptoms offer fruitful areas for cultural variations in health perceptions.

In conclusion, Japanese cultural patterns of activity and interpersonal relationships explain much of their desire to rely on self care. The Health Belief Model's explanatory variables of perceived efficacy of physician care, and perceived susceptibility to illness take different forms in the United States and Japan, although they are still related to self care actions. Cultural variations in levels of personal and relational autonomy in every day life styles and attitudes about the body are most critical in describing cross-national differences in health behavior. Although differences in the structure of health care delivery play a role, they take second place to the impact of the cultural milieux of the two industrial countries.

Acknowledgement-Support for this project was provided in part by National Institute on Aging, RO1-AGO5876.

\section{REFERENCES}

1. Levin L. S. and Idler E. L. The Hidden Health Care System: Mediating Structures and Medicine. Ballinger, Cambridge, 1981.

2. Williamson J. D. and Danaher K. Self Care in Health. Croom Helm, London, 1978.

3. Fleming G. V., Giachello A. L., Andersen R. M. and Andrade P. Self care: Substitute, supplement or stimulus for formal medical care services? Med. Care 22, 950, 1984.

4. Dean K. J. (I986) Self carc behavior: Implications for 
aging. In Self Care and Health in Old Age (Edited by Dean K., Hickey T. and Holstein B. E.), pp. 58-93. Croom Helm, Dover, NH.

5. Haug M. R., Wykle M. L. and Namazi K. H. Self-care among older adults. Soc. Sci. Med. 29, (2), 171-183, 1989.

6. Rakowski W., Hickey T. and Dengiz A. N. Consequence of health and treatment perceptions among older patients and providers of primary care. Int. J. Aging Hum. Dev. 25, (1), 63-77, 1987.

7. Levin L. S., Katz A. H. and Holst E. Self Care: Lay Initiatives in Health. Prodist, New York, 1976.

8. Hickey T. Health and Aging. Brooks/Cole, Monterey, CA, 1980

9. Becker M. H., Brachman R. H. and Kirscht J. P. A new approach to explaining sick role behavior in low-income populations. Am. J. Publ. Hlth 64, 205, 1974.

10. Janz N. K. and Becker M. H. The Health Belief Model: A decade later. Hith Educ. Q. 11, 1, 1984.

11. Caudill W. The cultural and interpersonal context of everyday health and illness in Japan and America. In Asian Medical Systems: A Comparative Study (Edited by Leslie C.), pp. 159-177. University of California Press, Berkeley, 1976.

12. Triaudis H. G. and Breslin R. W. Cross-cultural psychology. Am. Psychol. 39, (9), 1006-1016, 1984.

13. Japan Ministry of Health and Welfare. Annual Report on Health and Welfare for 1989, October. Ministry of Health and Welfare, Tokyo, Japan, 1989.

14. Lock M. M. East Asian Medicine in Urban Japan. University of California Press, Berkeley, 1980.

15. Ohnuki-Tierney E. Illness and Cultural in Contemporary Japan. Cambridge University Press, London, 1984.

16. Ford A. B., Haug M. R., Jones P. K., Roy A. W. and Folmar S. J. Race-related differences among elderly urban residents: A cohort study, 1975-1984. J. Geront. 45, (4), S163-S171, 1990.

17. Haug M. R. and Lavin B. Consumerism in Medicine. Sage, Beverly Hills, CA, 1983.

18. Krantz D. S., Baum A. and Wideman M. V. Assessment of preferences for self treatment and information in health care. J. Person. Soc. Psychol. 39, 977-990, 1980.

19. Haug M., Breslau N. and Folmar S. J. Coping resources and selective survival in mental health of the elderly. J. Geront. 2, (4), 468-491, 1989.
20. Berkman L. and Breslow L. Health and Ways of Living: The Alameda County Study. Oxford University Press, New York, 1983.

21. Duke University Center for the Study of Aging and Human Development. Multidimensional Functional Assessment: The OARS Methodology, 2nd edn. The Center for the Study of Aging and Human Development, Durham, NC, 1978.

22. Lock M. Cultural construction of the menopausal syndrome: The Japanese case. Maturitas 10, 317-332, 1988.

23. Coulton $C$. and Frost $A$. K. Use of social and health services by the elderly. J. Hlth Soc. Behav. 23, 330-339, 1982.

24. Steslicke W. E. The Japanese state of health: A politicaleconomic perspective. In Health, Illness, and Medical Care in Japan (Edited by Norbeck E. and Lock M.), pp. 24-65. University of Hawaii Press, Honolulu, 1987.

25. Powell M. and Anesaki M. Health Care in Japan. Routledge, London, 1990.

26. Lock M. M. Protests of a good wife and wise mother: The medicalization of distress in Japan. In Health, Illness, and Medical Care in Japan (Edited by Norbeck E. and Lock M.), pp. 1-23. University of Hawaii Press, Honolulu, 1987.

27. Japanese Ministry of Health and Welfare. Annual Report on Health and Welfare for 1983, October. Ministry of Health and Welfare, Tokyo, 1983.

28. Long S. O. Health care providers: Technology, policy, and professional dominance. In Health, Illness, and Medical Care in Japan (Edited by Norbeck E. and Lock M.), pp. 66-88. University of Hawaii Press, Honolulu, 1987.

29. Antonovsky A. A model to explain visits to the doctor: With specific reference to the case of Israel. J. Hlth Soc. Behav. 13, 446-454, 1972.

30. Sonoda K. Health and Illness in Changing Japanese Society. University of Tokyo Press, Tokyo, 1988.

31. Long S. O. The ins and outs of doctor-patient relations in Japan. Am. J. Chinese Med. VIII, (1), 37-46, 1980.

32. Long S. O. and Long B. D. Curable cancers and fatal ulcers: Attitudes toward cancer in Japan. Soc. Sci. Med. 16, 2101-2108, 1982.

33. Hakuhodo Institute of Life and Living. Changing Lifestyles in Japan 4: Japanese Seniors, Pioneers in the Era of Aging Populations. Tokyo, Japan, 1987. 


\section{APPENDIX}

\section{Symptom List}

These symptoms are grouped by possible type of classification, but will be put in random order in the questionnaire. For each symptom, respondents will be asked if it was experienced in the past four months, and if so (1) what action was taken in response, and (2) if $s /$ he considered it mild, fairly serious or very serious. Data from prior work of the investigators are included, as evidence of subjects' understanding of the symptoms as an illness.

\begin{tabular}{|c|c|c|c|}
\hline & $\begin{array}{l}\text { Number of } \\
\text { persons } \\
\text { reporting } \\
\text { symptom }\end{array}$ & $\begin{array}{l}\% \text { using some } \\
\text { form of self } \\
\text { care as first } \\
\text { response }\end{array}$ & $\begin{array}{l}\% \text { considering } \\
\text { symptom fairly } \\
\text { or very serious }\end{array}$ \\
\hline \multicolumn{4}{|l|}{ Digestive system: } \\
\hline Diarrhea for more than one day & 28 & 86 & 43 \\
\hline Abdominal pains, for at least a couple of days & 77 & 94 & 36 \\
\hline Repeated indigestion or upset stomach & 105 & 98 & 19 \\
\hline Trouble moving bowels regularly & 118 & 100 & 16 \\
\hline \multicolumn{4}{|l|}{ Heart and circulation: } \\
\hline Repeated pains in or near the heart & 70 & 90 & 40 \\
\hline Shortness of breath, even after light work & 162 & 80 & 26 \\
\hline Swelling of feet, legs or hands & 198 & 82 & 20 \\
\hline Dizziness & 124 & 84 & 14 \\
\hline \multicolumn{4}{|l|}{ Muscular-skeletal system: } \\
\hline Pain or swelling in any joints or muscles during the day & 191 & 82 & 29 \\
\hline Waking up with stiff or aching joints or muscles & 260 & 90 & 23 \\
\hline Frequent backaches & 179 & 95 & 32 \\
\hline \multicolumn{4}{|l|}{ Infections: } \\
\hline Sore throat or running nose with a fever as high as $100^{\circ} \mathrm{F}$ for at least two days & 61 & 94 & 43 \\
\hline A cough anytime during night or day which lasted for several weeks & 103 & 82 & 34 \\
\hline Any infections, irritations or pain in the ears & 62 & 79 & 29 \\
\hline Any infections, irritations or pain in the eyes & 116 & 84 & 27 \\
\hline Sneezing for two weeks or more & 46 & 52 & 6 \\
\hline \multicolumn{4}{|l|}{ General tiredness: } \\
\hline Feeling tired for weeks at a time for no special reasons & 84 & 82 & 33 \\
\hline Sudden feelings of weakness or fainting & 144 & 100 & 25 \\
\hline Getting up some mornings tired and exhausted even with the usual amount of rest & 261 & 78 & 14 \\
\hline \multicolumn{4}{|l|}{ Not otherwise classified: } \\
\hline Frequent headaches & 88 & 88 & 38 \\
\hline Unexpected bleeding from any part of the body & 57 & 82 & 30 \\
\hline Skin rash or breaking out on any part of the body & 119 & 84 & 19 \\
\hline A burning sensation when urinating & 30 & 73 & 17 \\
\hline Itching, bleeding or pain of the rectal area & 66 & 79 & 18 \\
\hline
\end{tabular}

'The balance either used 'nothing', followed by self care, or immediately visited a physician. 